\title{
Special Issue "Selected papers presented at the 2008 Spring Meeting of the Quantum Optics and Photonics Section of the German Physical Society"
}

\author{
M. Weidemüller • M. Fleischhauer
}

Published online: 28 March 2009

(C) Springer-Verlag 2009

The 2008 Spring Meeting of the German Physical Society, taking place at the newly constructed science and congress center Darmstadtium, was characterized by a lively exchange between the different areas of modern Atomic, Molecular and Optical Physics. The large number of joint sessions shared between the different Sections of the German Physical Society and six interdisciplinary symposia demonstrated this dedication to research beyond the classical borders of disciplines. The large number of oral and poster contributions within the Section Quantum Optics and Photonics itself, being one of the largest Sections within the German Physical Society, reflect the increasing tendency to broaden the scientific goals and to explore new crossroads between different fields. It was a pleasure to witness the high standards of the presentations, in particular the ones presented by enthusiastic diploma and doctoral students.

For this special issue we tried to convey a flavor of the wealth of presentations within the Quantum Optics and Photonics Section. The invited contributions, which all underwent the well-established refereeing process of Applied Physics B, were chosen on the basis of recommendations by the session chairs of the Spring Meeting. It was our in- tention to cover a large variety of subjects, systems and processes. Therefore, the topics of this Special Issue range from fundamental questions in quantum optics to applications in metrology and medical research. The articles address a broad variety of systems such as trapped ions, Bose-Einstein condensates, ultracold molecules, solitons in photorefractive materials, photoelectrons, ultrashort laser pulses, and the human breath. The processes under investigation include atoms coupled to high-finesse cavities, coherence in adiabatic transfer and diffusion, entanglement in molecular dissociation, nonlinear effects in photonic materials, and the interactions of atoms with strong laser fields. In the spirit of supporting young researchers, most of the principal investigators of the articles in this Special Issue are at an early stage of their career.

We thank the local organizers of the Darmstadt meeting, in particular our colleagues Gernot Alber, Gerhard Birkl and Thomas Walther, for realizing such an excellent conference. This special issue has been made possible by the professional support of Editor-in-chief Frank Träger and of Jutta Kaisig from the University of Kassel whom we also thank for her help.
M. Weidemüller $(\bowtie)$

Physikalisches Institut, Universität Heidelberg, Philosophenweg 12, 69117 Heidelberg, Germany

e-mail: weidemueller@physi.uni-heidelberg.de

\section{Fleischhauer}

Fachbereich Physik, Universität Kaiserslautern, Erwin-Schrödinger-Str., 67663 Kaiserslautern, Germany e-mail: mfleisch@physik.uni-kl.de 